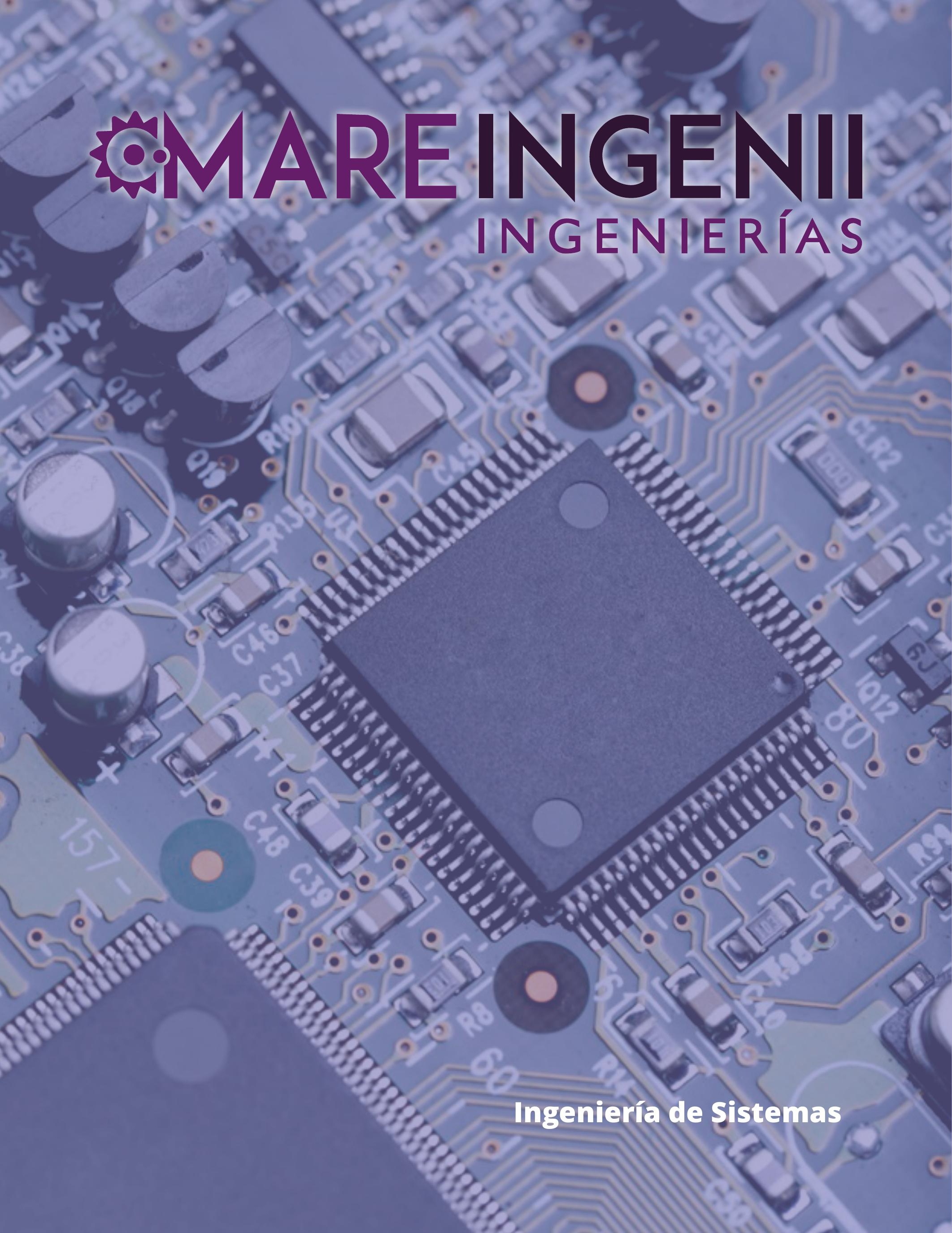




\title{
La importancia de un diseño instruccional para la gestión educativa de personas con deficiencia auditiva en el aprendizaje en línea
}

\author{
The value of an instructional design for the educational \\ management of people with hearing impairment in e-learning
}

Francy Yolanda Martínez Pinzón'

${ }^{1}$ Ingeniera y magíster en Administración y Planificación Educativa. Correo electrónico: franyoly1@yahoo.es

Como citar: Martínez Pinzón, F. 2019. La importancia de un diseño instruccional para la gestión educativa de personas con deficiencia auditiva. Mare Ingenii. Ingenierías 1(1). Disponible en http:// cipres.sanmateo.edu.co/index.php/mi

Recibido: Marzo 12 de 2019

Aceptado: Agosto 18 de 2019

\section{RESUMEN}

Este artículo determina la importancia de un diseño instruccional, dirigida a personas con deficiencia auditiva, facilitando el desarrollo de las actividades que se abordan en el proceso de enseñanza-aprendizaje en línea. Para tal fin, la observación se llevó a cabo con un estudiante que está desarrollando este proceso formativo, en la cual se analizaron los diferentes factores que afectan la gestión educativa del docente, en cuanto a la asesoría que se les brinda en el aprendizaje en línea. Este análisis ayuda a establecer el mejoramiento en los protocolos de enseñanza y formación para que contribuyan a garantizar el acceso y la consolidación de la calidad educativa. Se establece como resultado la elaboración de una guía instruccional docente orientado a estudiantes con deficiencia auditiva en el aprendizaje en línea. Por último, se plantean las conclusiones que servirán de aporte para futuras investigaciones.

Palabras clave: diseño instruccional; gestión educativa; discapacidad; deficiencia auditiva; aprendizaje en línea.
ABSTRACT

This research aims to determine the importance of an instructional design, aimed at people with hearing impairment, facilitating the development of activities that are addressed in the online teachinglearning process. For this purpose, the observation was carried out with a student who is developing this training process, in which the different factors that affect the educational management of the teacher were analyzed in terms of the advice provided in online learning. This analysis helps to establish the improvement in teaching and training protocols so that they contribute to guarantee access and consolidation of educational quality. It is established as a result the preparation of a teaching instructional guide aimed at students with hearing impairment in e-learning. Finally the conclusions will serve as input for future research.

Keywords: instructional design; educational management; disability; hearing impairment; e-learning. 


\section{INTRODUCCIÓN}

La comunidad académica, así como la docencia universitaria, brindan valiosas oportunidades que motivan a plantearnos, continuamente, cómo aportar conocimiento a nuestro sistema de enseñanza-aprendizaje. Igualmente existe la pregunta de cómo ayudar a nuestros estudiantes a mejorar cada día su desempeño personal, laboral y profesional.

Es de allí que surge la necesidad de buscar herramientas que incentiven el aprendizaje en línea. Y puesto que es un nuevo escenario, que da soporte a la enseñanza y el aprendizaje, apoyándose en el uso de las Tecnologías de la Información y Comunicación (en adelante TIC), el rol del docente está más orientado a ser un facilitador y mediador del aprendizaje de sus estudiantes, en la medida que los lazos de comunicación se realizan en forma tanto sincrónica como asincrónica.

En este marco, su finalidad es determinar la importancia de un diseño instruccional, dirigida a personas con deficiencia auditiva, de manera que puedan acceder a ellas sin ningún tipo de barreras, facilitando el desarrollo de las actividades que se abordan en el proceso de enseñanza-aprendizaje en línea. Hace falta un diseño instruccional para la planeación del docente. Lo anterior orientado a la formación en línea con un enfoque hacia las necesidades educativas de aprendizaje; principalmente, los estudiantes con deficiencia auditiva, ya que la ausencia de estrategias metodológicas conlleva a que estos discentes se desmotiven o abandonen su proceso de formación.

La gestión educativa de calidad reúne elementos del enfoque sistémico, la administración y planeamiento estratégico, y la búsqueda del mejoramiento continuo, con el propósito de obtener mejores resultados educativos. Es decir, un alumno graduado con las competencias necesarias para desenvolverse en el mercado de trabajo, el diario vivir o continuar las especializaciones de estudios superiores.

\section{ESTADO DEL ARTE}

A renglón seguido, se presentan los antecedes históricos e investigativos más relevantes, que soportan este estudio.

\section{- Antecedentes históricos}

Los antecedentes en cuanto a la atención de la población con deficiencia auditiva, se debe a la evolución y desarrollo de las apuestas educativas, a la normatividad que se ha adoptado y a los lineamientos y orientaciones producidas con el trabajo sectorial necesario para la integración de todas las áreas y entes involucrados.

De acuerdo con Pérez [1], se afirma que la educación virtual tiene sus orígenes en los cursos a distancia por correspondencia en Inglaterra, en el año 1840, la cual fue evolucionando junto con el desarrollo de la tecnología. Se cita la creación de la radio, la televisión, la computadora, los recursos multimediales, el internet y los artefactos digitales (laptops, iphones, ipads o teléfonos inteligentes) que hacen que la educación virtual y el uso de las TIC sean más comunes en la vida diaria del ser humano.

Barberá, Romiszowski, Sangrá y Simonson [2] describen que a finales de los años 1980, y principios de 1990, el desarrollo de sistemas de comunicación de fibra óptica permitió la expansión en el ámbito educativo de sistemas de audio, de video en vivo, y de alta calidad. Entre los años 1997 al 2008, el concepto de discapacidad se había fortalecido, debido al fuerte movimiento que generaron las propias personas discapacitadas en Europa, Norteamérica y, por supuesto en América Latina, con el apoyo de la Organización de las Naciones Unidas (ONU) y la Organización de las Naciones Unidas para la Educación, la Ciencia y la Cultura (Unesco) [3].

A finales del siglo $X X$, surgen dos géneros de traducción audiovisual: la subtitulación para personas que presentan algún tipo de deficiencia auditiva y la audio-descripción para aquellas que han desarrollado una deficiencia visual. Estos dos géneros reciben el nombre de "accesibilidad", puesto que dan respuestas a las necesidades de esta población. El nacimiento de estos géneros determina la conmemoración del Año Internacional del Deficiente (1981) y el Año Europeo de las personas con discapacidad (2003), involucrando los medios audiovisuales. De ahí que se regularan y publicaran normativas sobre el tema', las mismas que se empezarán a formar en niveles de enseñanza reglada, entre los especialistas en accesibilidad [4].

\footnotetext{
${ }^{1}$ Como la norma UNE 153010 del año 2003 sobre el subtitulado para sordos a través del teletexto. También
} 
Más adelante, a partir de los años 1990, se promueve la necesidad de integración social o inclusividad. Hicieron énfasis en los principios de igualdad y derechos humanos para todas las personas, con la introducción de las TIC en los establecimientos educativos [5]. En Colombia, desde el año 2001, el Ministerio de Tecnologías de la Información y las Comunicaciones (Mintic), en alianza con la Federación Nacional de Sordos de Colombia (Fenascol), han evolucionado con respecto a la apropiación de las tecnologías de la información y la comunicación según el centro de relevo [6].

La herramienta de apropiación de las TIC ofrece contenidos y espacios donde el lenguaje de señas y la lengua escrita prevalecen en el acceso a la información, el aprendizaje, la comprensión, la construcción de conocimientos y, sobre todo, en la motivación al uso de las TIC en personas sordas, siendo no solo consumidores; sino productores de información, revolucionando así su inmersión en la red.

Hacia el año 2008, Daniela Galindo [7] dio a conocer el software para romper barreras de comunicación, a través de la página llamada Hablando con Julis. La motivación al crear este software fue el deseo de mejorar la calidad de vida de su hermana, quien presenta discapacidades físicas y mentales. Este software emplea palabras representadas por imágenes. Cada término tiene una descripción escrita y hablada. Asimismo, incluye también un video que despliega la expresión de la palabra usando el lenguaje de signos.

Por otro lado, la comunicación es la base del desarrollo de todo ser humano. Es lo que permite aprender, socializar, tener amigos, estudiar, trabajar y ser parte activa de la sociedad. El modelo pedagógico de Hablando con Julis permite avanzar en la comunicación, lectura y escritura con total facilidad. Este software va dirigido a personas desde los tres (3) hasta los 85 años de edad que presentan algún tipo de discapacidad, como trastorno espectro autista, dificultades en el habla, sordos, dificultades para leer y escribir, parálisis cerebral, síndrome de Down, personas en condición de analfabetismo y personas iniciando su proceso para hablar, leer y/o escribir.

Según Mintic [8], en Colombia, con la implementación del Plan Vive Digital del año 2010, se establece un plan tecnológico que tiene como objetivo la masificación del uso del internet. A través de este se han conectado niños, emprendedores, empleados públicos, personas en condición de discapacidad, campesinos, industriales y a pequeños y medianos empresarios. Con este plan, el uso de las TIC se ha convertido en una herramienta esencial para el desarrollo del país.

En su versión final de la propuesta Modelo de adaptación en ambientes virtuales de aprendizaje para personas con discapacidad, Lancheros, Carrillo y Lara [9] proponen implementar herramientas educativas que consideren el perfil y la caracterización del estudiante. Lo anterior, para determinar su estilo de aprendizaje, ya sea verbal o visual. Así, de acuerdo con este desplegar la información que más se ajuste a sus características, que le permitirían a un estudiante con discapacidad mejorar sus procesos de aprendizaje.

El Instituto Colombiano para la Evaluación de la Educación (ICFES), diseñó una prueba electrónica para que, por primera vez, las personas con discapacidad auditiva puedan presentar las pruebas SABER $11^{\circ}$ por computador. Quienes desarrollaron la prueba el día 14 de septiembre de 2014, señalan en la plataforma sus respuestas sin el uso de lápiz ni papel.

Además, el Instituto Nacional para Sordos (INSOR) [10], explica que la alianza que efectuó dicha institución con la Universidad Sergio Arboleda, a partir de mayo de 2017, será la encargada de capacitar, con énfasis en "Educación inclusiva para sordos", a los docentes que están en los programas académicos de especialización en educación, maestría en educación y maestría en educación para los derechos humanos. Esto la constituye en la primera universidad del país que impulsa programas académicos en educación superior, a nivel de postgrado, orientados al desarrollo pedagógico y a la formación de profesionales cualificados para la atención educativa a la población sorda.

\section{- Antecedentes investigativos}

Un primer trabajo de investigación corresponde al de Bernal [11], denominado Estrategias de enseñanza para maestros con niños sordos, del Instituto nuestra señora de la Sabiduría, tiene como objetivo detallar las características de las estrategias que usan los docentes en el desarrollo de los contenidos con estudiantes sordos. Infiere que el docente decide, según los procesos de enseñanza, cuáles estrategias utilizar para motivar, apoyar los contenidos curriculares tales como organizadores previos, organizadores gráficos e ilustraciones. 
De la mano con el tema del anterior párrafo, González [12], en su trabajo que lleva por título Evaluación del diseño instruccional para cursos virtuales en la enseñanza de las matemáticas en Educación Superior, presenta como objetivo describir las características de los diseños instruccionales presentes en los cursos virtuales de las asignaturas relacionadas con matemáticas, en educación superior. Concluye que, para cumplir con éxito con los procesos de enseñanza aprendizaje (blended-learning o e-learning), es necesario dotar al estudiante y al docente de los conocimientos y herramientas necesarias para tal propósito. Así, es vital que el maestro tenga unas bases pedagógicas que le permitan elegir dentro de los recursos en red y dentro de las actividades que ofrecen las plataformas.

Por tanto, sugiere que la formación docente definitivamente debe existir dentro de las entidades educativas con una cobertura total de sus maestros, generando espacios de cómo alcanzar los objetivos propuestos para el aprendizaje, con elementos propios de un diseño instruccional, pero que contemple las fases de un diseño instruccional como el Addie. Además, agrega que el diseño instruccional que se implemente en una institución, debe ser un reflejo del modelo pedagógico que se quiere seguir. Por lo anterior, es importante socializar el propósito del modelo entre docentes, alumnos y con la comunidad en general.

En relación con lo ya mencionado, vale anotar que esta investigación es un aporte esencial en la implementación de un diseño instruccional que contenga la planeación metodológica y los protocolos de enseñanza, para orientar a las estudiantes que presentan necesidades de aprendizaje con deficiencia auditiva, y así lograr un buen desempeño académico por parte del educando. El docente debe realizar la planeación, el seguimiento, evaluación y control de estas actividades. Debe dar una respuesta oportuna a las inquietudes presentadas, manteniendo siempre el principio de la motivación, con el fin de mejorar su aprendizaje.

Así mismo, Castellano, González y Torroledo [13] realizaron un trabajo identificado con el nombre Gestión educativa para la atención de las dificultades específicas de aprendizaje en la básica primaria del Colegio Rural Quiba Alta (Bogotá). La estrategia de gestión se denomina "Educar para la diversidad", atendiendo las necesidades reales de la sociedad, generando transformaciones que favorezcan la calidad de vida y bienestar común desde la labor pedagógica. De lo allí dicho, concluyen que los instrumentos aplicados fueron pertinentes para identificar la necesidad prioritaria de atender las dificultades específicas de aprendizaje que afectan el nivel académico, social y emocional de los niños y niñas, desde diferentes áreas a nivel institucional.

Una de las recomendaciones de Castellano, González y Torroledo [13] es priorizar la atención de las dificultades específicas de aprendizaje para mejorar la calidad educativa en las instituciones distritales. Esto, por medio de la implementación de estrategias de gestión que involucren a la comunidad educativa.

En este sentido, se deduce la importancia de realizar una gestión educativa en pro de las necesidades de aprendizaje de las personas con deficiencia auditiva. Cabe recordar que "La revolución educativa" ha avanzado en el logro de la educación inclusiva. Da la posibilidad de acoger en los establecimientos educativos a todas las personas en los establecimientos educativos para acceder al uso de las TIC, atendiendo una de las metas que es la educación para todos, puesto que la educación es un derecho establecido en la constitución colombiana.

Se hace indispensable que, desde el momento de la matrícula, se realice la caracterización del estudiante que presenta algún tipo de deficiencia auditiva. De esta manera se puede orientar y hacer un seguimiento minucioso de su condición y su proceso formativo, dándole a conocer el contenido programático, el cronograma de entrega de actividades, el material de apoyo, las actividades y las evaluaciones a desarrollar.

Ruizy Dávila [14], en su Propuesta de buenas prácticas de educación virtual en el contexto universitario, plantean como propósito formular prácticas docentes para facilitar procesos formativos de calidad. Dichos procesos han sido fundamentos, clasificados en un proceso instruccional que incluye las fases de planificación didáctica previa al inicio de la formación, el desarrollo didáctico de la formación (con actividades de inicio y ejecución) y el cierre técnico y administrativo de dicho proceso.

Por lo expuesto en esta propuesta, concluyen que el e-learning, o educación virtual, canalizado por vía de aulas virtuales, es una modalidad educativa con un gran potencial para la formación en el contexto de la sociedad de la información y del conocimiento en general. En particular, de la universidad sobre la base de docentes implicados en la ejecución de buenas prácticas en el desarrollo de sus labores didácticas y tutoriales. 
Es necesario intensificar los esfuerzos en procura de una educación virtual de calidad, logrando un perfil de competencia adecuado, para contar con docentes idóneos de la modalidad. Esta propuesta incumbe a la investigación. Al hablar de educación virtual, o aprendizaje en línea, se considera un modelo que ha tenido un crecimiento muy significativo, comprendiéndose como un escenario de enseñanza y aprendizaje en el ciberespacio. En ella, el docente debe brindar las herramientas tecnológicas y metodológicas para que el estudiante se motive y se interese en el proceso de enseñanza-aprendizaje, teniendo la posibilidad de acceder desde cualquier área, sin tener que realizar ningún desplazamiento, desarrollando las habilidades y competencias en diferentes áreas del conocimiento. Asimismo por el hecho de que el estudiante pueda comunicarse, hacerse entender y comprender, teniendo seguridad en el entorno en que se está desenvolviendo.

En consecuencia y en relación con lo anterior, Duarte [15], en su trabajo denominado Propuesta de estrategias metodológicas para la enseñanza aprendizaje de la asignatura de Español en la Universidad Católica de Honduras, Nuestra Señora Reina de la Paz, Campus San Isidro, La Ceiba, concluye que hay una completa dependencia del texto y la realización de trabajos sugeridos. Además, hay un dominio bastante bajo en relación con la comprensión lectora.

Por esto, es necesario motivar a los alumnos a convertirse en lectores competentes, a través de actividades que permitan aprovechar el tiempo libre en espacios de lectura y escritura. También, de la misma manera, recomienda motivar a los docentes para que se mantengan en permanente capacitación, en relación con el uso de estrategias de enseñanza en el aula para lograr resultados importantes.

La citada propuesta se relaciona igual con esta investigación, debido a la innovación de nuevas estrategias metodológicas. Así, se espera que el estudiante con necesidades educativas de aprendizaje y deficiencia auditiva logre un mayor nivel de interactividad y flujos de comunicación, logrando los objetivos establecidos en el curso. De manera tal que él mismo deba apropiarse de nuevos conocimientos y experiencias, que le permitan resolver cualquier tipo de barrera que se pueda presentar. Es muy importante resaltar que para la comprensión de textos e interpretación de contenidos, se han de emplear descriptores visuales, pues las imágenes y videos deben llevar subtítulos y su respectiva descripción, para el óptimo desarrollo de las actividades de aprendizaje.

Por último, tenemos el trabajo de López [16], quien realizó la propuesta Modelo pedagógico pertinente a los estilos de aprendizaje de los estudiantes de pregrado con limitación auditiva que acceden a los cursos virtuales de la Universidad Nacional a distancia. Postula como objetivo describir el modelo pedagógico a los estilos de aprendizaje de los estudiantes de pregrado con deficiencia auditiva, para optimizar el proceso de aprendizaje en los cursos virtuales de la Universidad. Concluye que el diseño de nuevos ambientes de aprendizaje permite reconceptualizar la forma de enseñar y aprender, acorde con el desarrollo de la sociedad contemporánea. Además, el docente debe apoyarse en nuevos recursos y replantearse el empleo de otros ya existentes.

Una de las recomendaciones que hace este trabajo es la tener siempre en cuenta el estilo visual de aprendizaje que predomina en este grupo poblacional. Esto es, con la ayuda de estrategias definidas, como mapas conceptuales, mentales, videos, películas, esquemas, resúmenes, subrayado, foros de discusión o debate, para así enfrentar la lectura y asimilación de textos y módulos.

La anterior investigación aporta elementos que sirven como base para la elaboración de la propuesta. Gracias a la introducción de las TIC, esta considera una herramienta esencial para el aprendizaje de los estudiantes con deficiencia auditiva. Por ello, es fundamental la labor del docente como facilitador que brinda los recursos y medios tecnológicos, aplicando los protocolos de enseñanza que contribuyen con su desarrollo formativo, permitiendo la adquisición de nuevos conocimientos así como el desarrollo de las competencias dadas en el plan de estudio.

\section{FUNDAMENTACIÓN TEÓRICA}

Esta sección se desarrolla en cinco aspectos que fundamentan el soporte teórico de esta propuesta investigativa: diseño instruccional, estrategias metodológicas, gestión educativa, deficiencias auditivas y aprendizaje en línea.

\section{- Diseño instruccional}

El desarrollo de cursos en entornos virtuales es un proceso que involucra el diseño de estrategias metodológicas innovadoras y el uso de los 
recursos tecnológicos que utilizan los docentes en el proceso de enseñanza-aprendizaje. Para tener un mejor punto de vista acerca de ello, se hará una mención más específica en lo expuesto por Inciarte, en cuanto que:

El diseño instruccional es el cerebro dentro de una unidad curricular virtualizada o asignatura virtual, ya que permite guiar todos los procesos, además de controlar, fomentar y fortalecer de manera eficiente los aprendizajes significativos y el desarrollo autónomo del estudiante, teniendo presente que es el protagonista de su propia formación, es él quien, a través de esfuerzo y perseverancia va alcanzando sus metas [17, p. 7].

El estudiante es autónomo. Él es quien desarrolla las diferentes actividades, tareas y lecturas de cada sección de aprendizaje. Además, es quien se autorregula, debido a que él mismo es quien marca su propio ritmo de aprendizaje, según sus posibilidades de tiempo, distribuyendo la carga de trabajo a lo largo del tiempo, a fin de poder culminar el total de actividades dentro de los plazos previstos. Sin embargo, García y Pérez, describen que el diseño instruccional "es un proceso sistemático, planificado y bien estructurado, capaz de enseñar el conocimiento organizado y elaborado para facilitar el procesamiento de la información y el aprendizaje hacia los alumnos ${ }^{2 \prime}[18$, p. 5].

Conforme a lo expuesto por Inciarte [17], se especifican los siguientes elementos, que conforman el diseño instruccional:

- Introducción

- Justificación

- Propósito, objetivo, competencias, indicadores

- Contenido o saberes

- Estrategias Instruccionales y construccionales

- Recursos

- Estrategias de evaluación y autoevaluación

- Glosario

- Referencias bibliográficas

El diseñar un curso virtual requiere delinear espacios educativos para el logro de los objetivos, enseñando destrezas y conocimientos. De forma tal que faciliten el éxito aprendizaje del estudiante que presenta deficiencia auditiva, estableciendo el trabajo colaborativo, con el manejo y uso de recursos para que él realice la selección de información y su propio modelo de construcción del conocimiento. Por eso Romero señala que:
El diseño instruccional es necesario en cualquier modalidad, para organizar de una manera sistemática no solo la enseñanza sino también el aprendizaje. Los profundos cambios que se han producido a raíz de los avances tecnológicos no dejan a un lado la forma como se viene diseñando la instrucción; es por ello que los modelos instruccionales de hoy se caracterizan por ser procesos integrales y holísticos, dialécticos, creativos y flexibles [19, p. 1].

\section{- Estrategias Metodológicas}

La introducción de las TIC hace que se mejore la calidad en el proceso de enseñanza-aprendizaje. Facilitaa los docentes los recursos tecnológicos para la aplicación de nuevas estrategias metodológicas. En lo que se refiere más específicamente a estrategias metodológicas Corrales, enuncia que:

Son acciones y/o actividades a realizar, los métodos didácticos y los medios que se requieren, con el fin de ajustarse a los conocimientos y capacidades del alumnado, conjugar todas las dimensiones competenciales implicadas con los contenidos asociados, presentar una lógica y coherencia al proceso, potenciar ciertos puntos para que el aprendizaje sea significativo y favorecer la participación del alumnado en su propio proceso den enseñanza-aprendizaje [20, p. 157].

La metodología, por tanto, permitirá a los maestros tomar decisiones sobre cómo enseñar. Permitirá preguntarse qué papel va a desempeñar el alumno, cuáles van a ser las funciones del maestro, cómo se van a desarrollar las tareas, en qué tiempos y espacios, con qué materiales y cuál será el proceso evaluativo llevado a cabo.

\section{- Gestión educativa}

La gestión educativa está orientada a fortalecer el Proyecto Educativo Institucional (PEI), partiendo de que cada centro educativo vela por la autonomía organizacional, con el objetivo de mejorar los procesos pedagógicos, contribuyendo a satisfacer las necesidades educativas de una comunidad. En el desarrollo de este marco conceptual, encontramos que Fernández, define la gestión educativa como "un paradigma que permite a los centros educativos y a las unidades administrativas, en las diferentes instancias de la gestión, orientar de forma permanente o continuada su funcionamiento hacia la mejora de resultados" [21, p. 107].

2 Resaltado por la autora. 


\section{- Deficiencias auditivas}

En lo que respecta al uso del vocablo en cuestión, Méndez, señala que "los términos deficiencia auditiva, sordera e hipoacusia, se utilizan para referirse a cualquier grado de pérdida de la capacidad para oír" [22, p. 175]. En lo que se refiere al vocablo empleado para hablar de una persona que tiene alguna deficiencia o discapacidad auditiva, Díaz et al. (2000, p. 35), argumentan que: "Términos como sordos, minusválidos, deficientes auditivos, discapacitados auditivos son inadecuados y que es más conveniente hablar de personas con dificultades auditivas, personas con deficiencias auditivas, personas con discapacidad auditiva o de personas sordas" [23, p. 35].

\section{- Aprendizaje en Línea o e-Learning}

La educación virtual o educación en línea hace referencia al desarrollo de programas de formación, tomando el ciberespacio como escenario de enseñanza y aprendizaje. También se le conoce con el nombre de e-learning o electronic learning (aprendizaje electrónico). Para ampliar este concepto, tomando como referente a Zambrano, quien expone al respecto:

Se entiende por aprendizaje virtual aquella estrategia que utiliza los principios y fines de una educación que debe brindarse a cualquier persona, independientemente del espacio y del tiempo gracias al uso de las $\mathrm{TIC}$, de materiales educativos y de diferentes elementos principales, medios y formas de comunicación [24, p. 16].

En la actualidad, muchas universidades en el mundo han adoptado el aprendizaje en línea, involucrando las TIC. Debido a su interactividad y operabilidad con los ambientes virtuales, algunas de ellas ofrecen modalidad mixta (presencial-virtual), en las cuales los estudiantes complementan el proceso formativo, puesto que ellos pueden acceder desde cualquier lugar, de acuerdo con su disponibilidad de tiempo.

La web es un medio en donde se puede realizar búsqueda de información, a través de la exploración de tutoriales, hipertextos, documentos html y pdf, videos, imágenes estáticas y animadas, que sirven como apoyo para el proceso educativo. También se pueden destacar otros elementos que contribuyen con la comprensión, análisis y la construcción del conocimiento, como son los simuladores, aplicaciones multimediales, juegos interactivos y las enciclopedias.

\section{METODOLOGÍA}

Para explicar este contexto, se hace referencia en lo expuesto por Arias, a saber, "el diseño de investigación es la estrategia general que adopta el investigador para responder al problema planteado" [25, p. 26]. Por eso, Gómez, establece que:

El término "diseño" se refiere al plan o estrategia concebida para obtener la información que se desee, es decir, es el plan de acción a seguir en el trabajo de campo [...] En el momento en que se diseña una investigación, depende del enfoque elegido y del planteamiento del problema [26, p. 85].

Una vez entendido el concepto de diseño, la investigación se desarrolla en lo no experimental. Con esta técnica se busca observar la situación que viven las personas que presentan deficiencias auditivas en su proceso de aprendizaje. Por eso, se hace necesario buscar herramientas que contribuyan a la inclusión y la igualdad de oportunidades, sin intervenir en la situación que les está afectando directamente. Lo anterior se hace esto con el interés de generar nuevos protocolos de enseñanza, adaptados para el aprendizaje en línea. Dichas herramientas apoyarán a los docentes en su metodología de enseñanza y a los estudiantes en su proceso de aprendizaje de manera mutua.

Igualmente, y como un complemento necesario, se hace referencia a lo expuesto por Toro y Parra en cuanto a que:

La investigación no experimental es aquella que se realiza sin manipular deliberadamente variables. Es decir, es investigación donde no hacemos variar internacionalmente las variables independientes. Lo que hacemos en la investigación no experimental es observar fenómenos tal y como se dan en su contexto natural, para después analizarlos [27, p. 158].

Es importante aclarar que al ser una investigación mixta, se alternan varias técnicas. Por ello, aparte de la encuesta, se aplica de igual manera la entrevista, solamente a un estudiante clave, que fue detectado en el aprendizaje en línea con deficiencia auditiva. Para comenzar esta sección, De la Puente, postula que "un instrumento de recolección es el cuestionario o test, a través del cual vamos a recoger la información sobre la unidad de observación" [28, p. 189]. Así mismo, tenemos a Moreno y Gallardo, quienes consideran que:

Un instrumento de medición adecuado es aquel que registra datos observables que representan 
verdaderamente a los conceptos o variables que el investigador tiene en mente. En investigaciones de campo, tanto cuantitativas como cualitativas, el investigador requiere utilizar instrumentos apropiados para que la información que obtenga sea válida [29, p. 47].

En la tabla 1, se resumen los instrumentos de recolección de datos que se emplearon para realizar la presente investigación. situación. Para la elaboración de la entrevista se plantearon igualmente once (11) preguntas. Por añadidura, aportando a este propósito, tomamos el planteamiento de Arias quien señala que:

La entrevista, más que un simple interrogatorio, es una técnica basada en un diálogo o conversación "cara a cara" entre el entrevistador y el entrevistado acerca de un tema previamente determinado, de tal manera que el entrevistador pueda obtener la información requerida [25, p. 73].

En esta investigación, el instrumento fue sometido a validación de los expertos, determinando la pertinencia y claridad de cada uno de los ítems que lo conforman. La validez se obtuvo a través de, por un lado, la validez de contenido, es decir, los ítems del instrumento responden a los objetivos propuestos.

Tabla 1. Instrumentos de recolección de datos. Fuente: elaboración propia.

\begin{tabular}{lccc}
\hline \hline Población Objetivo & Instrumentos & $\begin{array}{c}\text { No. } \\
\text { Preguntas }\end{array}$ & $\begin{array}{c}\text { Tipo de } \\
\text { datos }\end{array}$ \\
\hline $\begin{array}{l}\text { Docentes } \\
\begin{array}{l}\text { Estudiante con } \\
\text { deficiencia auditiva }\end{array}\end{array}$ & Encuesta & 11 & $\begin{array}{c}\text { Cuantitativos } \\
\text { Eualitativos }\end{array}$ \\
\hline \hline
\end{tabular}

En primer lugar, se utiliza como técnica la encuesta, distribuida en once (11) preguntas cerradas con respuestas múltiples (preguntas con varias alternativas). Lo anterior, para recopilar los datos acerca de la labor del docente que orienta el aprendizaje en línea y que deber servir de base para generar una posible alternativa de solución a esta. En lo que se refiere a este aspecto más concretamente, Grasso enuncia que:

La encuesta es un procedimiento que permite explorar cuestiones que hacen a la subjetividad $y$, al mismo tiempo, obtener esa información de un número considerable de personas... Así mismo, la encuesta permite obtener datos de manera más sistemática que otros procedimientos de observación [30, p. 13].

La investigación contempla, en un segundo lugar, la entrevista para recolectar la información precisa. Esta va enfocada a aquel estudiante con deficiencia auditiva, lo que permite establecer las falencias que tiene el aprendizaje en línea para mejorar esta
Por otro lado, a través de la validez de construcción que son los ítems del mismo formulados en relación con los indicadores de la tabla. De acuerdo con los postulados de Landeau, se puede argumentar de la mano del autor que:

La validez es el grado en que el instrumento proporciona datos que reflejen realmente los aspectos que interesan estudiar. La confiabilidad es el grado con el cual el instrumento prueba su consistencia, por los resultados que produce al aplicarlo repetidamente al objeto de estudio [31, p. 81].

Asimismo, en relación con el coeficiente de confiabilidad Medina y Verdejo, comentan que este "indica el grado de la relación entre los conjuntos de puntuaciones que representan medidas de la misma característica. Hay diferentes métodos para estimar el coeficiente de confiabilidad de un instrumento dependiendo del propósito y su uso particular" [32, p. 74]. 
Partiendo de esta referencia para valorar la fiabilidad de las medidas, se usó el coeficiente alfa de Cronbach. La aplicación del instrumento se sometió a un pequeño grupo de docentes pertenecientes a la población en estudio. Con los resultados obtenidos, se determinó la confiabilidad del instrumento y su aplicación a la muestra en estudio. En relación con este elemento, se tomaron los siguientes datos de la encuesta para calcular el coeficiente Alfa de Cronbach.
- Se llevó a cabo la tabulación y las respectivas gráficas (circulares), reflejando así los porcentajes hallados.

Así, considerando el carácter de esta investigación, se realizó un análisis detallado de los datos obtenidos para cada interrogante de la encuesta, mediante la elaboración de tablas de distribución de frecuencias.

Tabla 2.Matriz para el cálculo del coeficiente Alfa de Croncbach. Fuente: elaboración propia.

\begin{tabular}{|c|c|c|c|c|c|c|c|c|c|c|c|c|c|}
\hline Elemento & $\mathrm{S}_{\mathrm{i}}^{2}$ & Item 1 it & em 21 & tem 3 & Item 4 & Item 5 & Item $6 \mathrm{H}$ & & Item 8 & & Item 10 & tem 11 & $s_{t}^{2}$ \\
\hline a & & 4 & 12 & 16 & 10 & 17 & 14 & 14 & 6 & 2 & 18 & 20 & 133 \\
\hline b & & 5 & 5 & 2 & 12 & 6 & 2 & 4 & 5 & 3 & 8 & 5 & 57 \\
\hline c & & 3 & 9 & 12 & 7 & 4 & 10 & 2 & 4 & 1 & 10 & 12 & 74 \\
\hline d & & 8 & 8 & 9 & & 8 & 12 & 1 & 12 & 14 & 17 & 14 & 108 \\
\hline & 199,88 & 3,50 & 6,25 & 26,19 & 7,25 & 24,69 & 20,75 & 26,69 & 9,69 & 27,50 & 18,69 & 28,69 & 871 \\
\hline
\end{tabular}

\section{ANÁLISIS DE LOS RESULTADOS}

En lo que atañe al análisis de los datos, encontramos lo que nos plantea Muñoz, "se refiere a la forma de utilizar la estadística para poder interpretar los antecedentes. Es la agrupación de datos en rangos significativos conforme a una selección adecuada que resulte en una interpretación útil para el investigador" [33, p. 84]. De acuerdo con los argumentos anteriormente expresados, se detalla el análisis de los datos recopilados en la investigación, de la siguiente manera.

- Se realizó la encuesta a veinte (20) docentes que participan en el proceso del aprendizaje en línea, profesionales de áreas técnicas y transversales.

- Se efectuó la entrevista al estudiante con deficiencia auditiva que presenta necesidades educativas en el aprendizaje en línea, quien cursa actualmente segundo semestre de Ingeniería de Sistemas y tiene 30 años.
Dichas frecuencias describen detalladamente las categorías, el cálculo del porcentaje de cada categoría, el porcentaje válido y el porcentaje acumulado, que constituye lo que aumenta en cada categoría de manera porcentual y progresiva. De ahí que a continuación se describen los ítems que se realizaron en la encuesta a docentes con los siguientes interrogantes.

- ¿De los siguientes elementos, indique cuáles aplicarían a un diseño instruccional?

De acuerdo con los resultados obtenidos, hay que realizar un plan de formación docente basado en los elementos esenciales que conforman la creación de un diseño instruccional para, de esta forma, contribuir con las necesidades de aprendizaje de los estudiantes que presentan deficiencia auditiva. 
Figura 1. Elementos que aplicarían a un diseño instruccional, según su porcentaje. Fuente: elaboración propia.

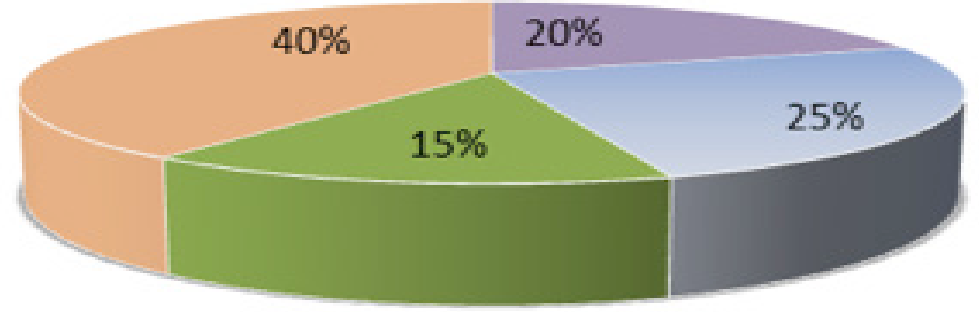

= Objetivos, secuenciación, métodos y evaluación

= Objetivos, contenidos, temporalización y evaluación

\footnotetext{
= Objetivos, contenidos, métodos y evaluación = No sabe/No responde (NS/NR)
}

Figura 2. Tipos de contenidos digitales como apoyo a la labor docente, según su porcentaje. Fuente: elaboración propia.

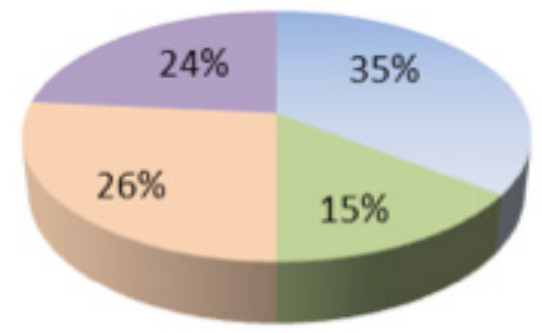

m Imagen y video $\quad$ Audio $\quad$ Texto $=$ Otros

- ¿Qué tipo de contenidos digitales utiliza como apoyo a la labor docente?

En relación con lo anterior, se evidencia que el manejo de diferentes herramientas que facilitan el aprendizaje y la comprensión de los contenidos es primordial. Estos son medios de expresión y aprendizaje que propician espacios de construcción del conocimiento y producción de contenidos. Además, desarrollan la creatividad e incentivan la investigación para así contribuir a mejorar la calidad educativa. 
- ¿Qué tipo de contenidos digitales ha creado que faciliten el desarrollo de las actividades de aprendizaje?

En consecuencia, se evidencia que parte del material que los docentes colocan en plataformas virtuales es generado por ellos mismos. Lo anterior puede favorecer el aprendizaje. Al ser creado por los profesores, es concebido teniendo en cuenta las particularidades del estudiante y los resultados del proceso enseñanza-aprendizaje que se pretenden obtener.
- ¿Qué programa emplea para la elaboración de contenidos digitales?

Se evidencia cómo la mayoría de los docentes utiliza Power point, y aunque es un programa muy usado para realizar presentaciones en diapositivas, con animación de texto e imágenes, hoy día existen otras herramientas. Lo anterior indica que el docente debería tener un mayor empoderamiento en el aprendizaje de las TIC, combinando habilidades, saberes y estrategias digitales que propicien ambientes de aprendizaje distintos y creativos.

Figura 3. Contenidos digitales que facilitan el desarrollo de las actividades de aprendizaje, según su porcentaje. Fuente: elaboración propia.

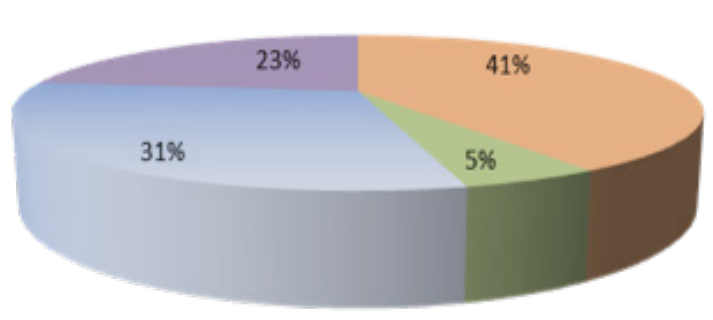

Guias

Libros

Presentaciones

n Videos

Figura 4. Programas elaboración de contenidos digitales, según su porcentaje. Fuente: elaboración propia.

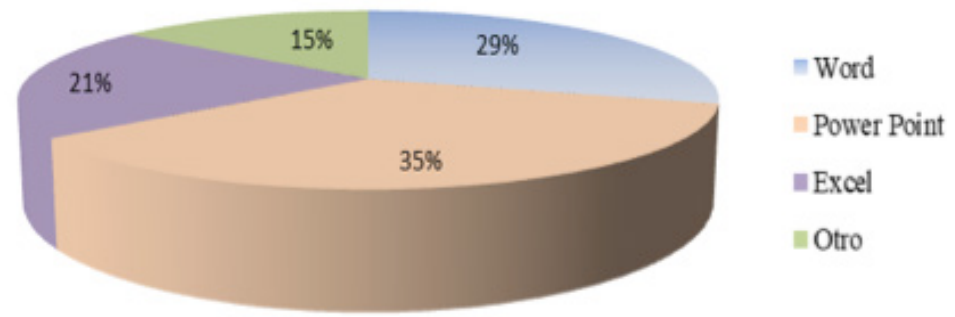


- ¿Qué aspectos considera esenciales para la elaboración de contenidos digitales?

Como se ha dicho ya, en un contenido digital, si bien es importante la calidad y el desarrollo, lo más significativo es que esa herramienta sea efectiva para el aprendizaje, que los contenidos se puedan modificar y actualizar con facilidad. Además debe tener un gran impacto visual y una mejor posibilidad de interacción, en donde el estudiante pueda buscar y seleccionar la información, para la construcción de su propio conocimiento.
¿Cuál de estos recursos educativos digitales emplea en el proceso de enseñanzaaprendizaje?

Conviene subrayar que el docente de hoy utiliza diferentes recursos didácticos, como apoyo en el proceso de enseñanza-aprendizaje. Esto facilita la interpretación de contenidos para que el estudiante desarrolle las actividades asignadas y prevalezca la participación activa y dinámica. Dichos recursos permiten realizar la búsqueda de información a través de buscadores especializados, enciclopedias online y desarrollar presentaciones online.

Figura 5. Aspectos esenciales para la elaboración de contenidos digitales, según su porcentaje. Fuente: elaboración propia.
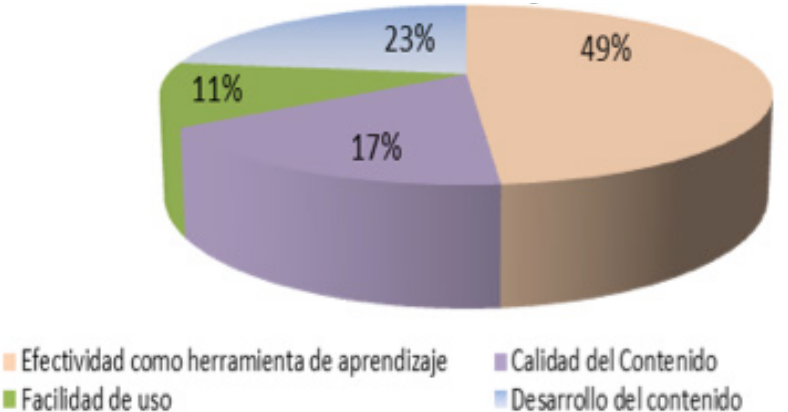

Figura 6. Recursos educativos digitales empleados en el proceso de enseñanza-aprendizaje, según su porcentaje. Fuente: elaboración propia.

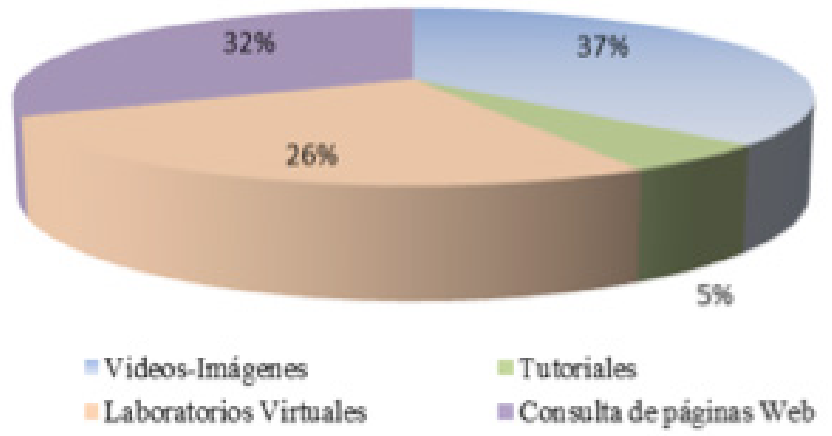


- ¿En su labor como docente ha tenido que manejar estudiantes con deficiencia auditiva?

Se evidencia que son muy pocos los docentes que han orientado procesos formativos a estudiantes con deficiencias auditivas. Asimismo, aparece la necesidad de que las instituciones de educación superior deban velar por el derecho a la educación de esta población.
- ¿ ¿Cuál de las siguientes estrategias metodológicas utiliza para la orientación del aprendizaje de un estudiante con deficiencia auditiva?

Es necesario implementar estrategias metodológicas, a pesar de que más del $44 \%$ de la población no han capacitado estudiantes con deficiencia auditiva. El porcentaje restante han utilizado estrategias metodológicas como implementación de tutoriales y videos, tutorías personalizadas, orientaciones con la ayuda de un intérprete, hablar de frente al estudiante, colocar su mano en la garganta con la asesoría de un intérprete.

Figura 7. No. De estudiantes con deficiencia auditiva, según su porcentaje. Fuente: elaboración propia.

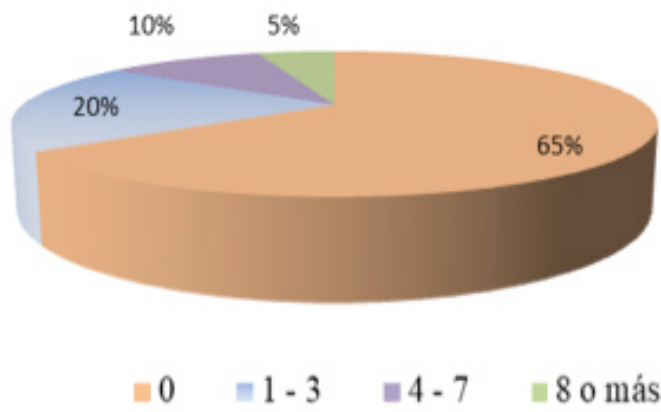

Figura 8. Estrategias metodológicas para la orientación del aprendizaje de un estudiante con deficiencia auditiva, según su porcentaje. Fuente: elaboración propia.

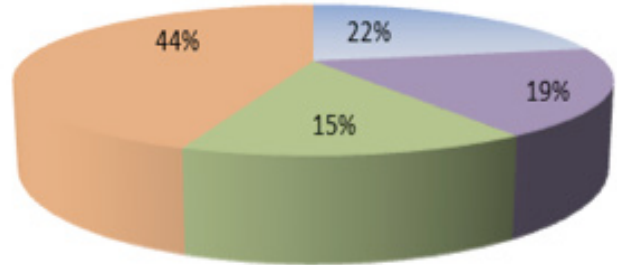
Mapas conceptuales
- Linea de Tiempo Resolución de problemas
* No sabe/No responde (NS/NR) 
- ¿Qué tipo de formación ha recibido para la orientación al estudiante con deficiencia auditiva?

Como resultado, tenemos que la mayoría de los docentes que están en el aprendizaje en línea han desarrollado contenidos digitales. Sin embargo, desconocen las pautas para elaborar dirigidos a personas que tienen necesidades educativas. Por lo tanto, se quiere fortalecer la conceptualización del diseño instruccional, con el fin de crear contenidos digitales para esta población.
- ¿Sobre qué tema le gustaría recibir capacitación para orientar a los estudiantes que presentan necesidades de aprendizaje con deficiencia auditiva?

Con respecto a la capacitación que les gustaría recibir a los docentes para atender a los estudiantes que presentan necesidades de aprendizaje con deficiencia auditiva, un 34\% manifestaron que sería indispensable conocer el manejo de herramientas tecnológicas, seguido del 32\% que desean conocer las pautas para la elaboración de contenidos digitales, 19\% desean conocer las orientaciones pedagógicas, y el 15\% restante requieren capacitación en las formas de comunicación. Así, es necesario brindar capacitación y desarrollar la guía para crear contenido digital que oriente a las personas que presentan necesidades educativas con deficiencia auditiva.

Figura 9. Capacitación orientación de estudiantes con deficiencia auditiva, según su porcentaje. Fuente: elaboración propia.

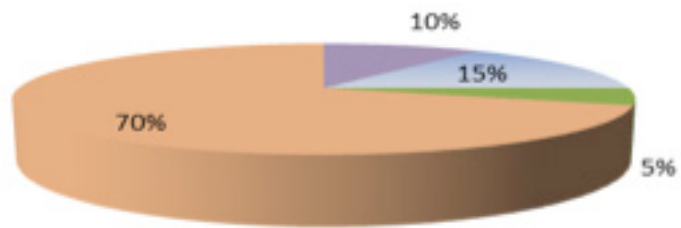

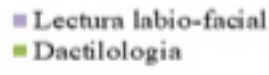

\author{
- Diseño instruccional contenidos digitales \\ Ninguna
}

Figura 10. Temática para orientar a los estudiantes que presentan necesidades de aprendizaje con deficiencia auditiva, según su porcentaje. Fuente: elaboración propia.

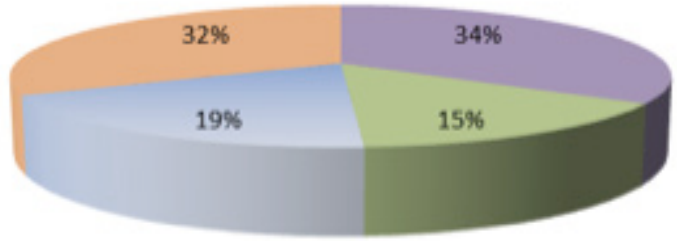

= Herramientas Tecnológicas

= Orientaciones Pedagógicas
= Formas de comunicación

"Elaboración de contenidos digitales 
- ¿Para qué cree que se deba implementar el diseño de un modelo para la gestión educativa de los estudiantes con deficiencia auditiva?

De este hallazgo, y del planteamiento citado, se deduce que es necesario crear un diseño instruccional para dar las pautas acerca de cómo crear material digital, para que los docentes elaboren y publiquen las guías de aprendizaje que orienten la formación pedagógica de los estudiantes que presentan necesidades educativas con deficiencia auditiva.

En la Tabla 3 se describe la matriz para el análisis de entrevista realizada al estudiante que presenta necesidades educativas con deficiencia auditiva en el aprendizaje en línea y su respectiva estructuración.

Figura 11. Diseño de un modelo para la gestión educativa de los estudiantes con deficiencia auditiva, según su porcentaje. Fuente: elaboración propia.

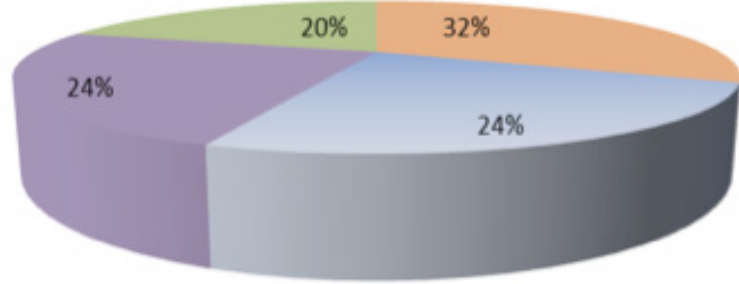

= Accedan a la información de forma fácil y cómoda

=Establezcan lazos de comunicación
- Ayudar a la adaptación del entorno

= Mejorar la calidad de vida

Tabla 3. Matriz para el análisis de entrevista a estudiantes. Fuente: elaboración propia.

\begin{tabular}{ll}
\hline \hline \multicolumn{1}{c}{ Categoría } & \multicolumn{1}{c}{ Subcategoría } \\
\hline Deficiencias Auditivas & - Origen \\
& - Lectura de Labios \\
& - Discriminación \\
& - Tipo de deficiencia \\
Estrategias Metodológicas & - Comprensión de contenidos \\
& - Organización de la información \\
Diseño Instruccional & - Medios de accesibilidad \\
& Audiovisual que entiende \\
& - Medios de accesibilidad \\
& audiovisual adecuados \\
Aprendizaje en Línea & Funcionamiento Plataforma \\
& virtual \\
& - Ayuda Plataforma Virtual \\
& Herramientas Virtuales \\
\hline \hline
\end{tabular}


A continuación se detalla cada una de las categorías planteadas para el análisis de la entrevista. Cabe resaltar que en la categoría de Deficiencias Auditivas, se identificaron los elementos de la Tabla 4.

Estos datos recolectados sirven como fundamento para la elaboración de material educativo, que sea claro y entendible. Esto, con el fin de mejorar los procesos comunicativos, aumentando la eficiencia y asertividad, para la adecuada construcción del conocimiento y la resolución de problemas.
Por otra parte, en la categoría Estrategias metodológicas (Tabla 5) se determinaron los siguientes elementos. El estudiante trata de memorizar y conceptualizar algunos contenidos para poder comprenderlos, le son muy útiles estas herramientas. Es necesario orientar a los estudiantes en la realización de sus actividades, brindando las estrategias metodológicas que favorezcan el proceso formativo, permitiendo la estructuración de frases y oraciones de fácil comprensión e interpretación, logrando la participación de los estudiantes en las actividades planteadas.

Tabla 4. Deficiencia auditiva. Fuente: elaboración propia.

\begin{tabular}{ll}
\hline \hline \multicolumn{1}{c}{ Subcategoría } & \multicolumn{1}{c}{ Unidad discursiva } \\
\hline Origen & $\begin{array}{l}\text { El estudiante manifestó haber perdido la } \\
\text { audición con el transcurrir del tiempo. } \\
\text { Por la necesidad de comunicarse y poder } \\
\text { expresar sus pensamientos e ideas aprendió a } \\
\text { manejar la lectura de los labios. }\end{array}$ \\
Elscriminación & $\begin{array}{l}\text { El estudiante tuvo una experiencia en } \\
\text { secundaria en donde fue discriminado por un } \\
\text { docente del área de Tecnología e } \\
\text { Informática, por usar un lenguaje muy } \\
\text { técnico, que era poco comprensible para él. } \\
\text { Inhabilidad auditiva, para manejar procesos } \\
\text { de aprendizaje. }\end{array}$ \\
\hline \hline
\end{tabular}

Tabla 5. Estrategias metodológicas. Fuente: elaboración propia.

\begin{tabular}{|c|c|}
\hline Subcategoría & Unidad discursiva \\
\hline $\begin{array}{l}\text { Comprensión de } \\
\text { contenidos }\end{array}$ & $\begin{array}{l}\text { La forma del objeto, es el descriptor } \\
\text { visual más llamativo para la } \\
\text { comprensión de contenidos }\end{array}$ \\
\hline $\begin{array}{l}\text { Organización de la } \\
\text { información }\end{array}$ & $\begin{array}{l}\text { Los mapas conceptuales y el gráfico de } \\
\text { líneas de tiempo, ofrecen una mejor } \\
\text { organización de la información. }\end{array}$ \\
\hline
\end{tabular}


Así mismo en la categoría Diseño Instruccional (Tabla 6), se tomaron los siguientes aspectos. Comenta que aunque faltan medios audiovisuales en la Universidad, se tiene una ventaja: el uso del correo institucional. Allí se informa de las actividades que se llevan a cabo y de las cuales pueden participar.
Para terminar, con la categoría Aprendizaje en línea (Tabla 7) se deduce que el docente debe brindar las herramientas virtuales que faciliten la comprensión e interpretación de los contenidos programáticos de las diferentes asignaturas que conforman su plan curricular, supliendo las necesidades de aprendizaje del estudiante con deficiencia auditiva.

Tabla 6. Diseño instruccional. Fuente: elaboración propia.

\begin{tabular}{|c|c|}
\hline Subcategoría & Unidad discursiva \\
\hline $\begin{array}{l}\text { Medios de } \\
\text { accesibilidad } \\
\text { Audiovisual que } \\
\text { entiende }\end{array}$ & $\begin{array}{l}\text { Se demuestra que los subtítulos y la auto } \\
\text { descripción de videos son elementos fáciles } \\
\text { para la comprensión de contenidos. Al } \\
\text { estudiante le gustaría ver en la plataforma } \\
\text { las actividades que debe desarrollar en las } \\
\text { diferentes asignaturas. Estas estas ayudas, } \\
\text { serían un apoyo valioso para el proceso de } \\
\text { enseñanza-aprendizaje }\end{array}$ \\
\hline $\begin{array}{l}\text { Medios } \\
\text { accesibilidad } \\
\text { audiovisual } \\
\text { adecuados }\end{array}$ & $\begin{array}{l}\text { En el momento que el estudiante se } \\
\text { matriculó manifestó su caracterización } \\
\text { como persona que presenta deficiencia } \\
\text { auditiva, con el fin de que le colaboraran en } \\
\text { todo lo concerniente a su proceso formativo. }\end{array}$ \\
\hline
\end{tabular}

Tabla 7. Aprendizaje en línea. Fuente: elaboración propia.

\begin{tabular}{|c|c|}
\hline Subcategoría & Unidad discursiva \\
\hline $\begin{array}{l}\text { Funcionamiento } \quad y \\
\text { ayuda en } \\
\text { plataforma virtual }\end{array}$ & $\begin{array}{l}\text { El estudiante requiere de asesoría } \\
\text { frecuente para el manejo de la plataforma } \\
\text { en la oficina de orientación y atención al } \\
\text { estudiante, ya que la plataforma presenta } \\
\text { videos que no contienen subtítulos ni auto } \\
\text { descripción, y que no alcanza a interpretar } \\
\text { ni entender. }\end{array}$ \\
\hline $\begin{array}{l}\text { Herramientas } \\
\text { Virtuales }\end{array}$ & $\begin{array}{l}\text { Las presentaciones visuales, tutoriales y } \\
\text { canales de video que tengan subtítulos son } \\
\text { las herramientas virtuales, que emplea en } \\
\text { su proceso formativo. }\end{array}$ \\
\hline
\end{tabular}




\section{CONCLUSIONES}

Al establecer la importancia de un diseño instruccional con estrategias metodológicas, se observa que los docentes utilizan recursos educativos tales como imágenes, videos, tutoriales, laboratorios virtuales y consulta de páginas web. Además, manejan programas como Word, PowerPoint, Excel, presentaciones, entre otros, para la elaboración de contenidos. No obstante, desconocen las pautas y protocolos de enseñanza para su aplicación y orientación que deben seguir en caso de tener una persona que presenta necesidades educativas con deficiencia auditiva.

De acuerdo a la investigación, se detectaron varios factores que se deben mejorar en el aprendizaje en línea. Se requiere del fortalecimiento del uso de herramientas tecnológicas para la elaboración de contenidos digitales que puedan orientar el aprendizaje de personas que presentan deficiencia auditiva.

Según los resultados arrojados por la investigación, los docentes desarrollan contenidos digitales en diferentes programas, realizan presentaciones. Sin embargo, falta incluir videos, un tipo de contenido digital y un descriptor visual, que ayude al aprendizaje de la persona con discapacidad auditiva.

Por todo esto, sería muy útil que, por medio de una guía instruccional, se pueda contribuir con el soporte en la gestión educativa del docente, la cual le brinde los protocolos de enseñanza necesarios para el desarrollo de material educativo que oriente a las personas con deficiencia auditiva.

Se sugiere incluir subtitulados en la elaboración de cualquier contenido educativo digital, combinando los conocimientos, habilidades y estrategias metodológicas que emplea el docente. Se debe propiciar un ambiente de aprendizaje creativo e innovador y así lograr una mayor cobertura de esta población, que puedan acceder, sin ninguna barrera, a la información que se le está suministrando en el aprendizaje en línea.

Además se recomienda la creación de una guía Instruccional que contenga los protocolos de enseñanza y recursos educativos que debe gestionar el docente, para el desarrollo de contenidos digitales, de manera exclusiva para las personas que presentan necesidades educativas, que se implementarán en el aprendizaje en línea.

\section{AGRADECIMIENTOS}

Quiero agradecer en primer lugar a Dios, por haberme guiado durante el lapso de esta investigación y ser mi aliciente para culminarla con éxito y doy gracias a mi familia por su apoyo y comprensión en todo momento, para llevar a feliz término este proyecto.

\section{LISTA DE REFERENCIAS}

[1] B. M. Pérez, Antología de competencias digitales. Implementación de la instrucción virtual en el sector educativo mexicano. México: Editorial unid, 2015.

[2] A. Barberá, A. Romiszowski, A. Sangrá, A. y M. Simonson, Educación abierta y a distancia. $1^{a}$. Ed. Barcelona, España: Editorial Uoc, 2006.

[3] G. E. Llinás, La orientación académica desde el bienestar universitario. Barranquilla, Colombia: Ediciones Uninorte, 2009.

[4] M. J. Alejandre, Buenas prácticas en la docencia universitaria con apoyo de Tic. Experiencias en 2015. 1ª . Ed. España: Servicio de Publicaciones Universidad de Zaragoza, 2015.

[5] P.S. Gento y F. R González, Integración educativa e inclusión de calidad en el tratamiento educativo de la diversidad. Madrid, España, 2010.

[6] Centroderelevo: Herramienta de apropiación Tic [Internet]. Colombia: Ministerio de Tecnologías de la información y las comunicaciones; c2018; [citado 14 de julio de 2019]. Disponible en: http://www.centroderelevo.gov.co/632/ w3-propertyvalue-15255.html.

[7] Hablandoconjulis, Discapacidad [Internet]. Colombia, c2008; [citado 2 de mayo de 2019]. Disponible en: http://www.hablandoconjulis.org/ softwarehcj

[8] Mintic: Avances [Internet]. Colombia: MinTIC; [Actualizado 21 de junio de 2019; citado 10 de agosto de 2019]. Disponible http://www.mintic. gov.co/portal/604/w3-propertyvalue-568.html

[9] D. Lancheros, A. Carrillo y J. Lara, Modelo de adaptación en ambientes virtuales de aprendizaje para personas con discapacidad; [Internet]. Colombia c2011. [Citado 21 de abril de 2019]. Disponible en http://www.bdigital.unal.edu. co/28835/1/26722-93648-1-PB.pdf. 
[10] INSOR, Por primera vez Colombia tendrá docentes con formación avanzada en educación para sordos [Internet]. Colombia: Instituto Nacional para sordos; 2017. [citado 16 de julio de 2019]. Disponible en: http://www.insor.gov.co/ home/por-primera-vez-colombia-tendra-programa-de-formacion-avanzada-en-educacion-para-sordos/

[11] A.L. Bernal, Estrategias de enseñanza para maestros con niños sordos, del Instituto Nuestra señora de la Sabiduría. Tesis. [Internet]. Colombia: Universidad Externando de Colombia, 2018. [citado 13 de agosto de 2019]. Disponible en https://bdigital.uexternado.edu.co/ bitstream/001/852/1/CCA-spa-2018-Estrategias_de_enseñanza_para_maestros_con_niños_sordos_del_Instituto_Nuestra_Señora_de_ la_Sabiduria.pdf

[12] V.A. González, Evaluación del diseño instruccional para cursos virtuales en la enseñanza de las matemáticas en Educación Superior. Tesis. [Internet]. Colombia: Tecnológico de Monterrey, 2016. [citado 13 de agosto de 2019]. Disponible en https://repositorio.tec.mx/bitstream/handle/11285/612456/Tesis\%20Maestria\%20Alberto.pdf?sequence $=1$

[13] L. A Castellanos, G. A. Guerrero y P. Y. Torroledo, Gestión educativa para la atención de las dificultades específicas de aprendizaje en la básica primaria del colegio rural Quiba Alta (Bogotá) [Internet], 2016. [citado 2 de enero de 2019]. Disponible en: http://repository.unilibre.edu. co/bitstream/handle/10901/8238/TESIS\%20 ABRIL\%2013\%20de\%202016\%20FINAL.pdf?sequence $=1$

[14] B. C Ruiz y A. A. Dávila, Propuesta de buenas prácticas de educación virtual en el contexto universitario [Internet], 2016. [citado 14 de agosto de 2019]. Disponible en: http://www. um.es/ead/red/49/bolivar_davila.pdf

[15] M. L. Duarte. Propuesta de estrategias metodológicas para la enseñanza aprendizaje de la asignatura de español en la Universidad Católica de Honduras, Nuestra Señora Reina de la Paz, Campus San Isidro, La Ceiba, Honduras [Internet], 2014. [citado 13 de agosto de 2019]. Disponible en: http://www.cervantesvirtual.com/ obra/propuesta-de-estrategias-metodologicas-para-la-ensenanza-aprendizaje-de-la-asignatura-de-espanol-en-la-universidad-catolica-de-honduras-nuestra-senora-reina-de-lapaz-campus-san-isidro-la-ceiba/
[16] M. L. López, Propuesta de modelo pedagógico pertinente a los estilos de aprendizaje de los estudiantes de pregrado con limitación auditiva (LA) que acceden a los cursos virtuales de la Unad. [Internet] Colombia: Universidad a distancia, 2013. [Citado 12 de agosto de 2019]. Disponible en: http://stadium. unad.edu.co/preview/UNAD.php?url=/bitstream/10596/1053/1/24216924.pdf

[17] R. M. Inciarte. Diseño instruccional por competencias para administrar unidades curriculares virtualizadas. Vol. 2, p. 7, 2009.

[18] S.N. García y D.C. Pérez. Creación de ambientes digitales de aprendizaje. México: Digital Unid, 2016

[19] N. A. Romero, Manual de diseño instruccional: una propuesta con tareas integradoras. 1 $^{\text {a }}$. Ed. México: Digital UNID, 2016.

[20] A. M. Corrales, Selección, elaboración adaptación y utilización de materiales, medios y recursos didácticos en formación profesional para el empleo. España: Ediciones Paraninfo, 2015.

[21] P. J. Fernández, La educación en República Dominicana: Retos y perspectivas: ensayos de 37 especialistas dominicanos. República Dominicana: Amigo del Hogar, 2002-

[22] E. Méndez, Introducción al estudio de la invalidez. 1ª. Ed. San José, Costa Rica: Universidad Estatal a Distancia, 1991.

[23] M. M. Díaz et al., Necesidades y problemas de la población con deficiencias auditivas en el proceso de integración en el mundo laboral. La realidad en Asturias. Oviedo, España: universidad de Oviedo. Servicio de publicaciones, 2000.

[24] W. Zambrano, Modelo de Aprendizaje Virtual para la educación superior. Maves basado en tecnologías Web 3.0. 1ª . Ed. Colombia: Ecoe Ediciones, 2012.

[25] F. G. Arias, El proyecto de investigación. Introducción a la metodología científica. $5^{a}$. Ed. Venezuela: editorial Episteme, 2006.

[26] M. M. Gómez, Introducción a la metodología de la investigación científica. $1^{\text {a }}$. Ed. Argentina: Brujas, 2006.

[27] I. D. Toro y R. D. Parra, Método y conocimiento. Metodología de la investigación. $1^{\text {a }}$. Ed. Colombia: Universidad EAFIT, 2006. 
[28] V. C De la Puente, Una guía para la investigación. 1'. Ed. Salamanca, Madrid: Editorial Complutense, 1995.

[29] A. Moreno y Y. Gallardo, Recolección de la información. Serie Aprender a Investigar. (3a. ed.). Colombia: Santafé de Bogotá, Arfoeditores Ltda., 1999.

[30] L. Grasso, Encuestas: elementos para su diseño y análisis. 1ª. Ed. Córdoba, Argentina: Brujas, 2006.

[31] R. Landeau, Elaboración de trabajos de Investigación. 1ª ed. Caracas, Venezuela: Alfa, 2007.

[32] D. M. Medina y C.A. Verdejo. Evaluación del aprendizaje estudiantil. $3^{a}$. Ed. San Juan, Puerto Rico: Isla Negra editores, 2001.

[33] R. C. Muñoz. Cómo elaborar y asesorar una investigación de tesis. 1a. Ed. Naucalpan de Juárez, México: Prentice Hall Hispanoamericana S.A., 1998. 